\title{
Optimización de la resistencia del cuero automotriz en operaciones de ensamble empleando un diseño experimental $3^{\mathrm{k}}$
}

\section{Optimization of the resistance of leather for automotive use in assembly operations using a $3^{\mathrm{k}}$ experimental design}

\author{
RUELAS-SANTOYO, Edgar Augusto †*, SÁNCHEZ-MÁRQUEZ, Juan Antonio', SÁNCHEZ-LUNA, \\ Antonio, CHACÓN-OLIVARES, Maria del Carmen
} \author{
México. 36820. (462) 6067900. \\ 'Universidad de Guanajuato. Guanajuato, México. \\ ID $1^{\text {er }}$ Autor: Edgar Augusto, Ruelas-Santoyo \\ ID $1^{\text {er }}$ Coautor: Juan Antonio, Sánchez-Márquez \\ ID $2^{\text {do }}$ Coautor: Antonio, Sánchez-Luna \\ ID $3^{\text {er }}$ Coautor: Maria del Carmen, Chacón-Olivares
}

Instituto Tecnológico superior de Irapuato. Carretera Irapuato - Silao km 12.5 Colonia El Copal. Irapuato, Guanajuato,

DOI: $10.35429 / J O T I .2019 .11 .3 .5 .13$

Recibido 7 de Julio, 2019; Aceptado 5 de Septiembre, 2019

\section{Resumen}

Dentro de la industria automotriz una de los materiales más utilizados para el confort del usuario es la piel animal, dicho material es utilizado para recubrir piezas que tendrán contacto con el consumidor. Uno de los problemas más importantes que se presentan con el manejo de la piel en operaciones de recubrimiento y ensamble dentro de plantas del sector automotriz es la falta de resistencia. Un tratamiento que se debe aplicar a la piel para mejorar sus condiciones de resistencia a la tensión mecánica, está en función de dos factores: porcentaje de humedad en la piel y cantidad de tensoactivo aplicado. En la presente investigación se establecen condiciones de operación óptimas para el manejo de la piel empleada en procesos del sector automotriz a partir de un diseño experimental factorial 3k. El caso de aplicación fue llevado a cabo en una empresa dedicada a fabricar descansabrazos para autos de gama alta. Los resultados de la investigación muestran una mejora del $97 \%$ en operaciones donde la resistencia de la piel es exigida para cumplir con los estándares de calidad establecidos en la industria automotriz.

\begin{abstract}
Resumen
Within the automotive industry one of the most used materials for user comfort is animal skin, this material is used to coat pieces that will have contact with the consumer. One of the most important problems that arise with the handling of the skin in operations of coating and assembly within plants of the automotive sector is the lack of resistance. A treatment that must be applied to the skin to improve its conditions of resistance to mechanical tension, is in function of two factors: percentage of moisture in the skin and amount of surfactant applied. In the present investigation, optimal operating conditions are established for the handling of the skin used in processes of the automotive sector based on a $3^{\mathrm{k}}$ factorial experimental design. The application case was carried out in a company dedicated to manufacture armrests for highend cars. The results of the investigation show an improvement of $97 \%$ in operations where the resistance of the skin is required to meet the quality standards established in the automotive industry.
\end{abstract}

Experimental design, leather, automotive industry

Diseño experimental, piel, industria Automotriz

Citación: RUELAS-SANTOYO, Edgar Augusto, SÁNCHEZ-MÁRQUEZ, Juan Antonio', SÁNCHEZ-LUNA, Antonio, CHACÓN-OLIVARES, Maria del Carmen . Optimización de la resistencia del cuero automotriz en operaciones de ensamble empleando un diseño experimental $3^{\mathrm{k}}$. Revista de Invención Técnica 2019. 3-11:5-13

*Correspondencia al Autor (Correo Electrónico: edruelas@itesi.edu.mx)

$\dagger$ Investigador contribuyendo como primer autor. 


\section{Introducción}

La industria automotriz es una de las más dinámicas y competitivas de México. En las últimas décadas, México ha llamado la atención de los principales actores del sector automotriz debido al crecimiento sostenido en la producción de vehículos y autopartes, así como a la fortaleza y las perspectivas de crecimiento de su mercado interno. Actualmente, México es el sexto productor más grande del mundo y la expectativa es que los próximos años el país avanzará varias posiciones. Este crecimiento es resultado de una combinación de factores, entre los que destacan la apertura de nuevas plantas de manufactura, la localización geográfica del país y el crecimiento de la industria en Estados Unidos, el principal destino de las exportaciones mexicanas de autopartes (Barrera y Pulido, 2016).

La importancia de la calidad de los productos es una prioridad fundamental para todas y cada una de las organizaciones que conforman el sector automotriz, es por ello que es primordial contar un seguimiento adecuado al proceso de fabricación y tener un control de las posibles causas que puedan afectar a calidad del producto (Chua, 2007).

Para mejorar la calidad y, en general para resolver problemas recurrentes y crónicos, es imprescindible apegarse a una metodología bien estructurada, para así llegar a las causas de fondo de cada uno de los problemas que realmente son importantes, y no quedarse en atacar efectos y síntomas (Gutiérrez, 2004).

El diseño de experimentos (DOE) por sus siglas en inglés, a través de experimentaciones y pruebas busca resolver los problemas que se presentan en los sistemas productivos. El diseño experimental consiste en determinar cuáles pruebas se deben realizar y de qué manera, para obtener datos que, al ser analizados estadísticamente, proporcionen evidencias objetivas que permitan responder las interrogantes planteadas, y de esa manera clarificar los aspectos inciertos de un proceso, resolver un problema o lograr mejorar u optimizar las operaciones (Montgomery, 2005).
El control de la calidad en la fabricación de curtidos precisa disponer de métodos de análisis y ensayos adecuados para examinar las materias primas, verificar los procesos de producción, vigilar las emisiones y sus tratamientos, y en definitiva para controlar la calidad del producto final. El ensayo del cuero terminado sirve para comprobar que posee calidad suficiente que su consumidor demanda. Para que los resultados del ensayo del cuero sean reproducibles en diferentes laboratorios es necesario unificar y normalizar estrictamente los ensayos de forma que en todos ellos se midan los mismos parámetros por los mismos procedimientos e instrumentos. La necesidad de la estandarización es especialmente acusada para los ensayos físicos, en primer lugar, por la irregular distribución de las propiedades físicas a lo largo del cuero y en segundo lugar por la considerable dependencia de los resultados respecto de las características del método utilizado. Es prácticamente imposible comparar resultados de ensayos físicos sino se ha seguido el mismo procedimiento (Font, 2002)

En el presente trabajo se propone analizar el efecto de los factores: cantidad de tensoactivo aplicado a la piel y el porcentaje de humedad presente en los cortes de piel utilizados en la fabricación de fundas para descansabrazos en la industria automotriz a partir de un diseño experimental factorial. El objetivo es aumentar la resistencia a la tensión y obtener los niveles óptimos de operación del enfundado para evitar defectos por rasgamiento y pérdidas de material, logrando así aumentar los indicares de producción y calidad en el sistema de producción.

La experimentación se llevó cabo dentro de la industria de fabricación de descansabrazos, el fin es poder para mejorar la condición física de la piel, específicamente la característica de calidad: resistencia a la tensión mecánica. El desarrollo de la investigación además del diseño experimental está basado en la metodología DMAIC ya que es un método estructurado para la solución de problemas. La metodología está compuesta de cinco fases interconectadas: definir, medir, analizar, mejorar y controlar (Carty, 2004).

Después de una breve introducción en la sección 1, el artículo se organiza de la siguiente forma: en la sección 1.1 se muestra la revisión bibliográfica llevada a cabo en la investigación. 
La sección 2 se compone por una descripción de los materiales y métodos utilizados. Los resultados de las experimentaciones y validación son mostrados en la sección 3. Por último, las conclusiones sobre la investigación propuesta conforman la sección 4

\section{Revisión Bibliográfica}

Los diseños de experimentos son muy utilizados dentro de la industria automotriz para realizar mejoras en los procesos de fabricación. El trabajo de (Suchana, 2017), propone una metodología de diseño para configuraciones de canales de refrigeración en moldes de inyección de plástico utilizados en el sector automotriz.

El trabajo presentado por (Tanco, 2018), analiza la soldadura láser aplicada para unir los laterales de un auto y su techo, la cual es realizada por medio de robots. Este proceso presentaba problemas de calidad, ya que la aparición de poros en ambos cordones de la soldadura es frecuente. Por ello, se recurrió a la experimentación y en concreto al diseño de experimentos (DOE) para mejorar el proceso. El proyecto obtuvo una reducción del $97 \%$ de los poros.

En el estudio llevado a cabo por (Romero, 2017), se realiza un diseño experimental para reducir la masa del pedal de freno sujeto a restricciones estructurales, esto debido a que los equipos FSAE a menudo reducían el factor de seguridad en métodos de diseño convencionales y subestimaban las cargas aplicadas a la pedalera. La parametrización del modelo geométrico del pedal de freno incluye un conjunto de ocho 8 variables de diseño, el DOE se genera usando el procedimiento denominado hipercubo latino. El resultado al que se llegó con el diseño fue encontrar la estrategia efectiva basada en metamodelos para minimizar la masa de un pedal de freno sin afectar el factor de seguridad. En (Villegas, 2013), se realizó un diseño de experimentos para determinar qué niveles de pintado de un producto minimiza el tiempo de espera del secado total, para ello realizó un experimento factorial completo de 3 factores con 2 niveles cada uno, los factores que se utilizaron fueron: tipo de pintura, método de secado y cantidad de capas que se aplican. Una vez realizado el análisis se conoció el método más efectivo para disminuir el tiempo de secado.
Dentro de la investigación presentada por (Li, 2017), se plantea que buscar la eficiencia del motor siempre es importante. Es por ello que propone un diseño de experimentos con cinco factores que ayudan a mejorar el rendimiento del motor. Se genera un diseño de experimentos $2^{5}$ para dicho análisis en donde arroja como resultado que todos los efectos son significativos para el rendimiento del motor. A través de la metodología de superficie de respuesta se genera la ecuación que permite encontrar los niveles óptimos de trabajo con el fin de obtener el mayor rendimiento del motor.

\section{Materiales y métodos}

Para llevar a cabo la investigación bajo un procedimiento ordenado se recurrió a la la metodología DMAIC, misma que es utiliza para llevar a cabo los proyectos Six Sigma con el fin de lograr la optimización de procesos. La metodología consta de cinco etapas: definir (D), medir (M), analizar (A), mejorar (M) y controlar (C). Las etapas son desarrolladas a lo largo de la sección de Materiales y métodos.

\section{Definir}

Uno de los procesos críticos en los que se hace uso de piel en el sector automotriz es la fabricación de partes tapizadas con el material, una parte muy importante es el descansabrazo ya que la pieza tiene contacto directo de forma física y visual con el usuario, de ahí la importancia de establecer condiciones de operación optimas que ofrezcan productos de calidad para el cliente a partir de un diseño experimental. En el caso de estudio, el proceso de tapizado de descansabrazos presenta un porcentaje de rechazos por encima del objetivo trazado dentro de la organización, mismos que son ocasionados por el desgarre de fundas al presentar poca resistencia en la piel. La piel desgarrada por falta de resistencia a la tensión ya no puede ser reprocesada teniendo la pérdida total del material (SCRAP), ver Figura 1. Con el fin de eliminar el desgarre de la piel, se plantea experimentar con los factores: \% de humedad y cantidad de tensoactivo, ya que ambos intervienen en el tratamiento y manejo de la piel en el sector automotriz. 


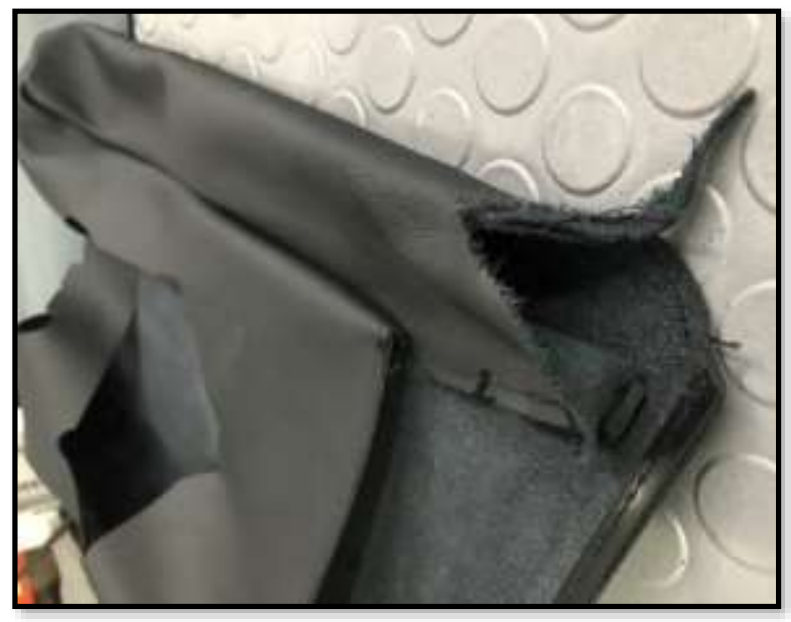

Figura 1 Funda Rasgada Fuente: Elaboración Propia

\section{Medir}

Uno de los indicadores de productividad más importantes en los sistemas productivos es el nivel de sigmas $(\sigma)$, el indicador hace alusión a la cantidad de piezas defectuosas por un fabricadas en cualquier operación presente en el proceso de manufactura, el ideal de cualquier sistema productivo es llegar a establecer un sistema $6 \sigma$ en el cual se generan únicamente de 3 a 4 defectos por millón. Para conocer el nivel de sigmas en operaciones de tapizado donde el principal problema es la rasgadura de la piel ocasionada por la falta de resistencia, es importante calcular la probabilidad de ocurrencia, para ello se analizó una muestra de 1000 piezas que se sometieron a la prueba de resistencia a la tensión. De acuerdo con (Montgomery y Runger, 2014), el tamaño de la muestra puede ser determinado a través de la Ecuación 1:

$\mathrm{n}=\frac{\mathrm{n}_{0}}{1+\frac{\left(\mathrm{n}_{0}+1\right)}{\mathrm{N}}}$

\section{Donde:}

\section{N: Tamaño de la población.}

$\mathrm{n}_{\mathrm{o}}$ : Tamaño de la muestra para una población finita

$\mathrm{n}_{\mathrm{o}}=\frac{\mathrm{z}_{\alpha / 2}^{2} * \mathrm{p} * \mathrm{q}}{\mathrm{e}^{2}}$

\section{p: proporción}

q: proporción complementaria (1- p)

e: margen de error z: nivel de confianza estandarizado.

El tamaño de la muestra mínimo queda definido en la Ecuación 2, tomando el tamaño de la población como el volumen de producción promedio mensual de 150 transformadores, el nivel de confianza empleado es del 95\% ( $\mathrm{z}=$ 1.96), una proporción del 50\%, y un margen de error del 3\%. El tamaño de muestra propuesto es más pequeño que el empleado, por lo tanto, el análisis estadístico es significativo y confiable.

$$
n=\frac{1,067}{1+\frac{(1,067+1)}{11,520}}=978 \text { muestras }
$$

De acuerdo al número de muestra calculado el realizar pruebas a un total de 1000 muestras de piel es significativo. Los resultados de las pruebas de tensión fueron de 745 piezas rasgadas $(74.5 \%)$. Obteniendo una probabilidad de que una muestra de piel no presente condiciones de rasgadura igual a $25.5 \%$. El nivel de simgas $(\sigma)$, es calculado a partir de la Ecuación 3.

Distribución normal inversa $\quad\left(e^{-\frac{d}{u}}\right)$

Empleando la Ecuación 3, se obtiene la ecuación 4 por sustitución:

Distribución normal inversa

$\left(e^{\frac{633}{1000}}\right)=0.077$ sigmas.

\section{Analizar}

La metodología seleccionada para lograr establecer parámetros de operación óptimos que permitan mejorar los indicadores de rechazos ocasionados por la falta de resistencia en la piel es la metodología de diseño de experimentos. Para comenzar con el diseño de experimentos, se realizaron cortes de piel estándar en sus medidas. En este diseño se analizó piel con grano medio en el grabado, la condición de grabado en la piel es de suma importancia ya que si se pierde el grano debido a los efectos de la humedad y tensoactivo se consideraría que no cumple con las especificaciones visuales del cliente, ver Figura 2 y 3 . Es por esa razón que se busca encontrar la combinación ideal entre tensoactivo y humedad sin perder el grabado en la piel y obtener una resistencia a la tensión adecuada. 


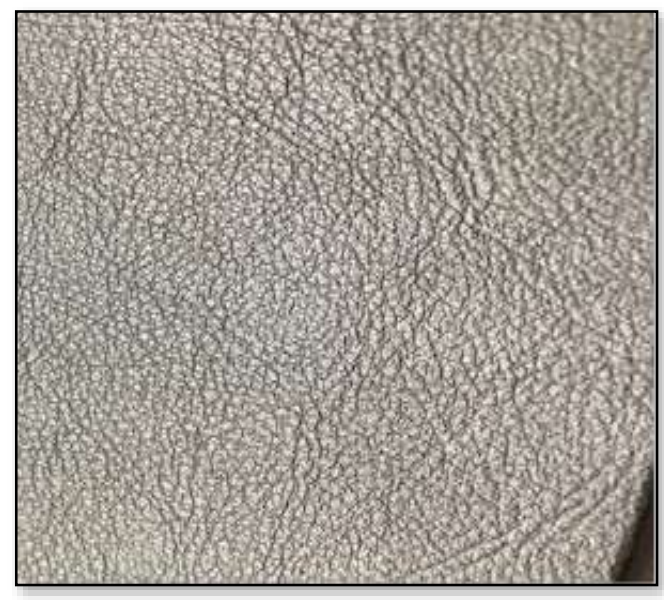

Figura 2 Piel con grano marcado

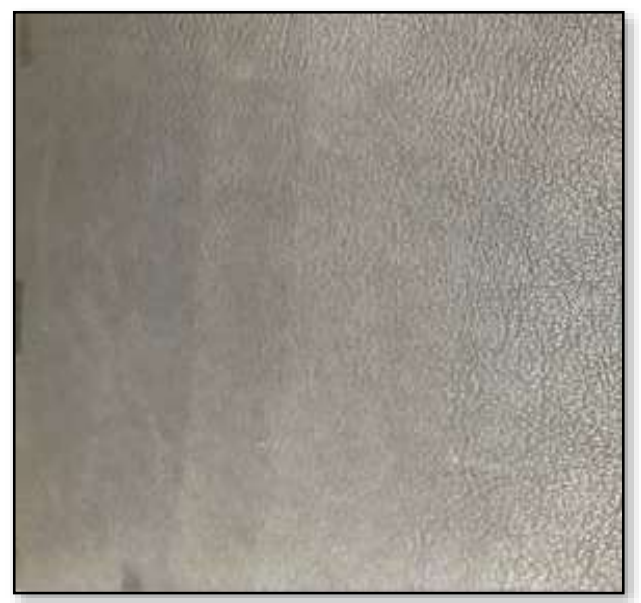

Figura 3 Piel con grano difuminado Fuente: Elaboración Propia

El objetivo de un diseño factorial es estudiar el efecto de varios factores sobre una variable de respuesta, cuando se tiene el mismo interés sobre todos los factores. Para llevar a cabo el análisis del efecto del tensoactivo y el \% de humedad sobre la piel se realizó un diseño de experimentos factorial $3^{\mathrm{k}}$, bajo la norma ISO 3376. Este diseño consiste en $3^{2}=9$ tratamientos, en donde el 3 representa los diferentes niveles a los que se fija cada factor en las diferentes pruebas, para este caso: alto, medio y bajo. El dos representa el número de factores que se requiere estudiar (cantidad de tensoactivo y \% de humedad) con la hipótesis que pueden afectar la variable de respuesta que en este caso es la resistencia a la tensión en la piel de uso automotriz. Al diseño experimental se agregó una réplica del diseño llevando a cabo un total de 18 tratamientos. La información general para realizar el diseño factorial se muestra en la Tabla 1.

\begin{tabular}{|c|c|c|c|}
\hline \multirow{2}{*}{ Factores } & \multicolumn{3}{c|}{ Niveles } \\
\cline { 2 - 4 } & $\mathbf{( - 1 )}$ & $(\mathbf{0})$ & $\mathbf{( 1 )}$ \\
\hline Tensoactivo (ml) & 50 & 62.5 & 75 \\
Humedad (\%) & 70 & 80 & 90 \\
\hline
\end{tabular}

Tabla 1 Factores para el análisis del diseño de experimentos factorial

\section{Fuente: Elaboración propia}

La cantidad de tensoactivo se probó a: $50,62.5$ y $75 \mathrm{ml}$. La humedad se controló por medio de una cámara climática donde se colocaron las muestras de piel por un lapso de 20 minutos a un porcentaje de humedad de $70,80 \mathrm{y}$ 90. Ver Figura 4. Para que la piel pueda ser liberada por parte del departamento de calidad debe pasar por inspección donde se realiza una prueba de resistencia, teniendo como valor de aceptación 130 Newtons, ver Figura 5.

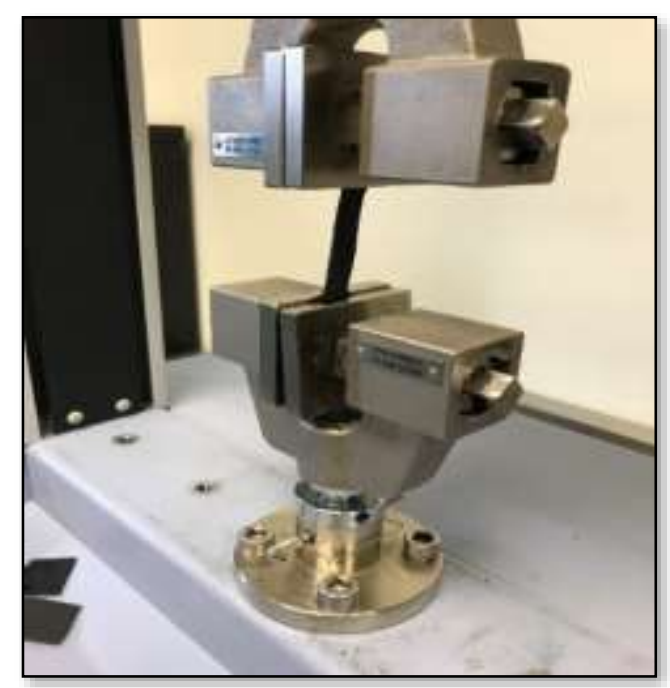

Figura 4 Control de humedad

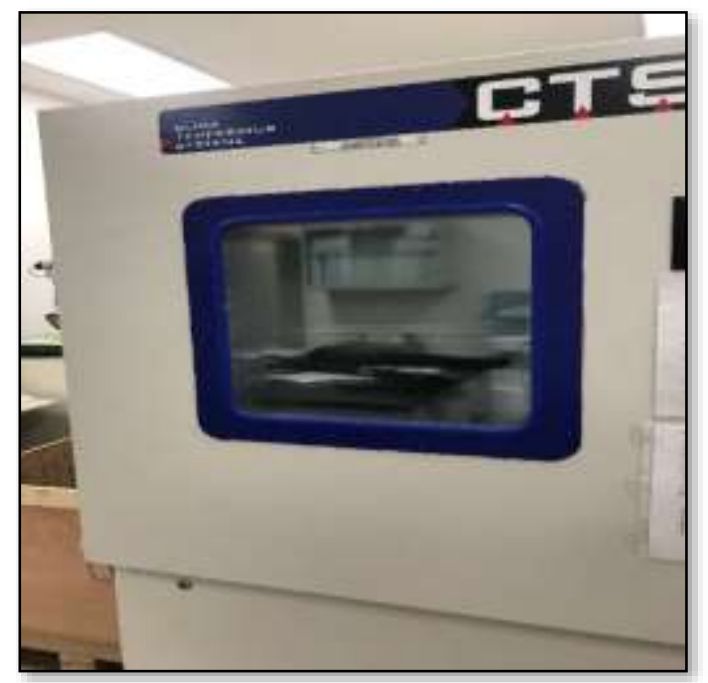

Figura 5 Prueba de resistencia en la piel Fuente: Elaboración Propia 
Las pruebas se realizaron siguiendo el orden del arreglo factorial $3^{2}$ con una réplica, el cual es un arreglo aleatorio con 18 tratamientos, Las pruebas realizadas se muestran en la Tabla 2.Los datos del experimento se analizaron a través del software Minitab 17

\begin{tabular}{|c|c|c|}
\hline Experimentación & $\begin{array}{l}\text { Cantidad } \\
\text { tensoactivo }\end{array}$ & e Humedad \\
\hline 5 & 62.5 & 80 \\
\hline 14 & 62.5 & 80 \\
\hline 4 & 50 & 80 \\
\hline 9 & 75 & 90 \\
\hline 16 & 50 & 90 \\
\hline 2 & 62.5 & 70 \\
\hline 3 & 75 & 70 \\
\hline 11 & 62.5 & 70 \\
\hline 6 & 75 & 80 \\
\hline 12 & 75 & 70 \\
\hline 17 & 62.5 & 90 \\
\hline 8 & 62.5 & 90 \\
\hline 15 & 75 & 80 \\
\hline 10 & 50 & 70 \\
\hline 7 & 50 & 90 \\
\hline 13 & 50 & 80 \\
\hline 1 & 50 & 70 \\
\hline 18 & 75 & 90 \\
\hline
\end{tabular}

Tabla 2 Matriz de diseño o arreglo factorial Fuente: Elaboración propia

\section{Resultados}

Una vez planificado el diseño experimental es preciso llevar a cabo cada uno de los tratamientos propuestos y registrar la variable de respuesta con el fin de analizar estadísticamente la variación de la resistencia en función de los factores involucrados en el diseño experimental propuesto, las respuestas a los tratamientos se pueden ver en la Tabla 3.

\begin{tabular}{|c|c|c|c|}
\hline Experimentasión & Contidad de temsosctivo (ma) & Humedod (N) & Resultado (N) \\
\hline 5 & 62.5 & 80 & 168.93 \\
\hline 14 & 62.5 & 80 & 167.30 \\
\hline 4 & 50 & 80 & 16240 \\
\hline 9 & 75 & 90 & 173.90 \\
\hline 16 & 50 & 90 & 163.50 \\
\hline 2 & 62.5 & 70 & 168.13 \\
\hline 3 & 75 & 70 & 172.13 \\
\hline 11 & 6.2 .5 & 70 & 168.30 \\
\hline 6 & 75 & 80 & 17230 \\
\hline 12 & 75 & 70 & 17120 \\
\hline 17 & 62.5 & 90 & 170 \\
\hline 8 & 62.5 & 90 & 17223 \\
\hline 15 & 75 & 80 & 173.30 \\
\hline 10 & so & 70 & 15980 \\
\hline 7 & 50 & 90 & 164.80 \\
\hline 13 & 50 & 80 & 16450 \\
\hline 1 & so & 70 & 160.10 \\
\hline 18 & 75 & 90 & 173.97 \\
\hline
\end{tabular}

Tabla 3 Resultado del efecto del tensoactivo y humedad sobre la resistencia de la piel Fuente: Elaboración propia

Se presenta el análisis de varianza (ANOVA) para determinar los efectos con mayor influencia sobre la variable de respuesta. La prueba del ANOVA demuestra que los factores principales; porcentaje de humedad y cantidad de tensoactivo, tienen un efecto significativo sobre la resistencia a la tensión de la piel ya que su valor-p es menor a 0.05 . Por otro lado, la interacción de ambos factores no tiene un efecto significativo sobre la variable de respuesta puesto que su valor-p es mayor que 0.05 , ver Tabla 4. El diseño experimentos presenta una $\mathrm{R}^{2}$ de $95.79 \%$ por lo que el modelo explica en buena proporción la variación generada por los factores y la variable de respuesta

\begin{tabular}{|l|c|c|c|c|c|c|}
\hline Fuente & $\begin{array}{c}\text { G } \\
\text { L }\end{array}$ & $\begin{array}{c}\text { SC } \\
\text { Sec. }\end{array}$ & $\begin{array}{c}\text { SC } \\
\text { Ajus. }\end{array}$ & \multicolumn{1}{c|}{$\begin{array}{c}\text { MC. } \\
\text { Ajusta } \\
\text { do }\end{array}$} & F & P \\
\hline Humedad & 2 & 29.276 & $\begin{array}{c}29.27 \\
6\end{array}$ & 14.638 & 16.76 & $\begin{array}{c}.00 \\
1\end{array}$ \\
\hline $\begin{array}{l}\text { Tensoacti } \\
\text { vo }\end{array}$ & 2 & $\begin{array}{c}326.12 \\
1\end{array}$ & $\begin{array}{c}32.12 \\
1\end{array}$ & 163.061 & $\begin{array}{c}186.7 \\
3\end{array}$ & $\begin{array}{c}.00 \\
0\end{array}$ \\
\hline $\begin{array}{l}\text { Humedad } \\
\text { Cantidad }\end{array}$ & 4 & 7.744 & 7.44 & 1.936 & 2.22 & $\begin{array}{c}.14 \\
8\end{array}$ \\
\hline Error & 9 & 7.859 & 7.859 & .813 & & \\
\hline Total & 17 & 371 & & & & \\
\hline
\end{tabular}

Tabla 4 Análisis de la varianza

Fuente: Elaboración Propia 
$\mathbf{R}^{2}=95.79 \%$ y $\mathbf{R}^{2}$ ajustado $=94.50 \%$

De acuerdo al análisis de la varianza el nivel de tensoactivo influye con mayor significancia en la resistencia de la piel que la humedad. La Figura 6, muestra la influencia de los efectos principales sobre la variable de respuesta en la cual se sugiera que en la búsqueda de mayor resistencia ambos factores deben ser fijados en niveles altos, es decir $90 \%$ de humedad y $75 \mathrm{ml}$ de tensoactivo. Sin embargo, también a mayor nivel de tensoactivo, la característica de calidad del grabado de la piel se pierde, ver Figura 3. En este caso trabajar con un nivel alto de tensoactivo no es funcional. Cabe señalar que no existe un efecto de curvatura significativo de los factores sobre la variable de respuesta. En la Figura 7, se muestra la superficie de respuesta de la resistencia a la tensión de la piel en relación a los factores involucrados en el estudio del diseño experimental.

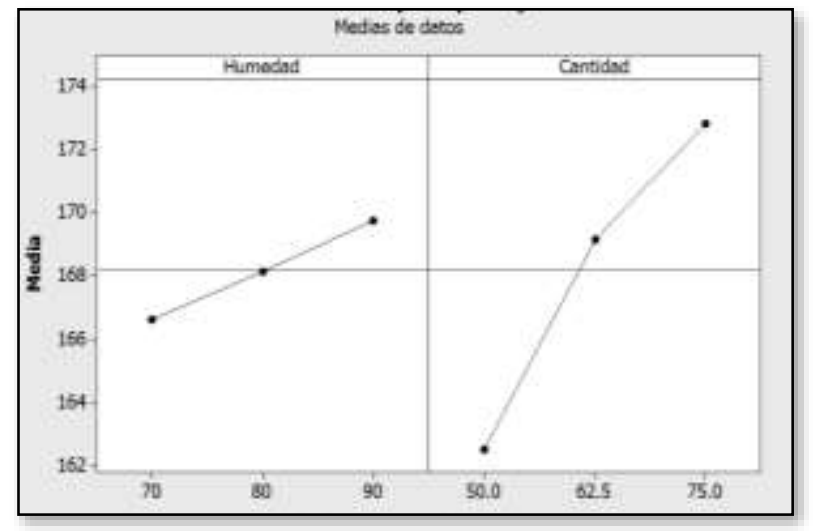

Figura 6 Control de humedad

Fuente: Elaboración propia en software Minitab

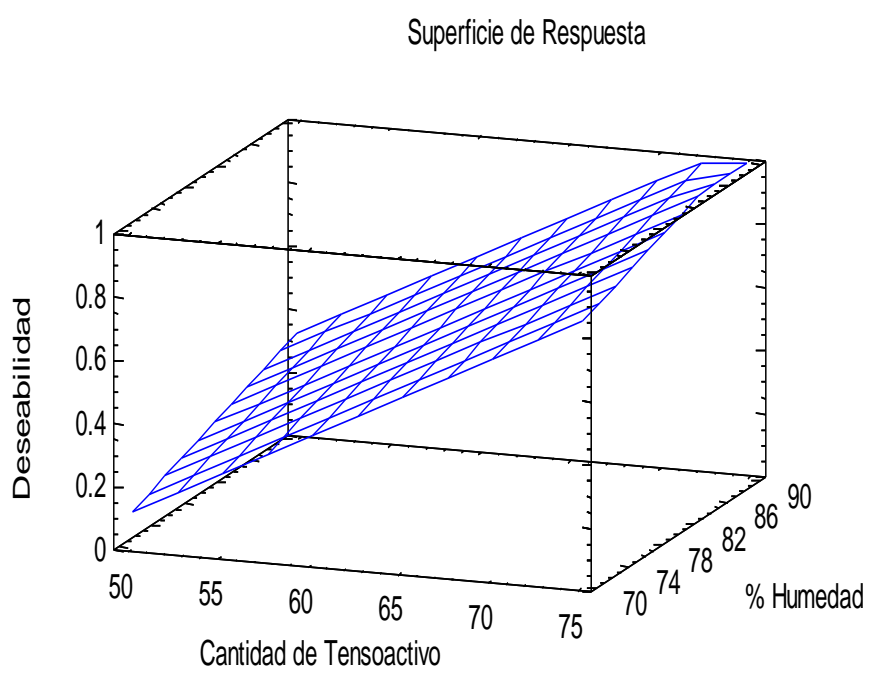

Figura 7 Control de humedad

Fuente: Elaboración propia en software Minitab
Para poder validar el diseño experimental realizado es vital cumplir con los supuestos de la metodología: normalidad, independencia y varianzas constantes de los residuales. Para comprobar el supuesto de normalidad se utilizó la prueba Anderson Darling, en la cual se debe cumplir la condición de $\mathrm{p}>0.05$, con el fin de concluir que los residuos se ajustan a una distribución normal. En este caso el valor p es igual a 0.250 por lo que se cumple el supuesto de normalidad, ver Figura 8. Para el supuesto de independencia se empleó el estadístico Durbin Watson en donde $\mathrm{D}<\mathrm{Du}$ para sugerir que no existe correlación. El valor calculado de $\mathrm{D}=$ 1.9711 y DU en tablas es igual 1.391, por lo tanto, no existe correlación entre los datos, ver Figura 9. La prueba de varianzas constantes se llevó a cabo por medio de la prueba de Bartlett, en donde el valor $\mathrm{p}$ debe ser mayor a .05 para cumplir con la condición, el valor p calculado es igual a 0.505 , por lo que se comprueba el supuesto de varianzas constantes, ver Figura 10.

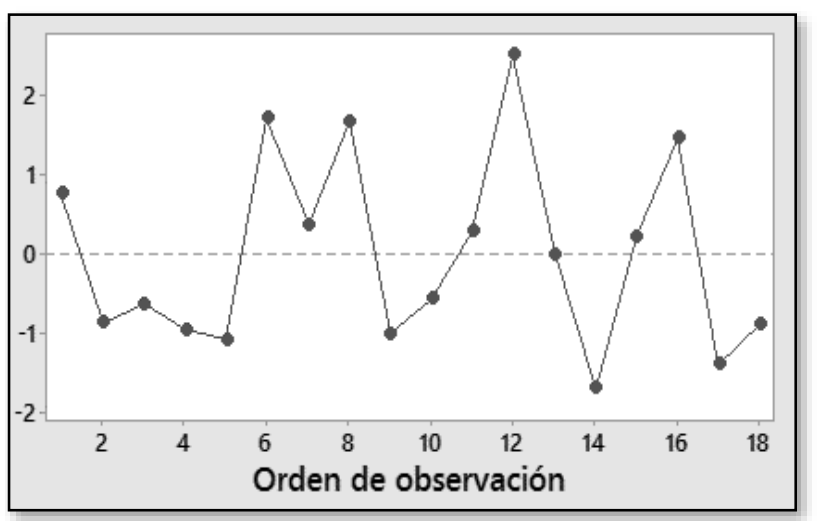

Figura 8 Prueba de Normalidad

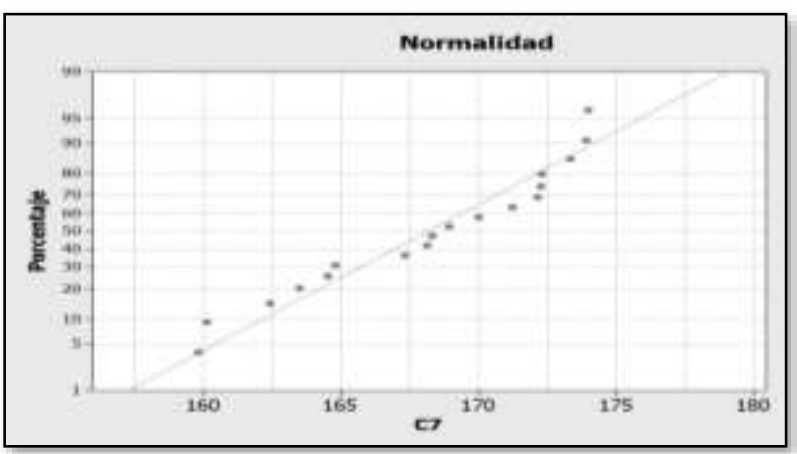

Figura 9 Independencia de datos 


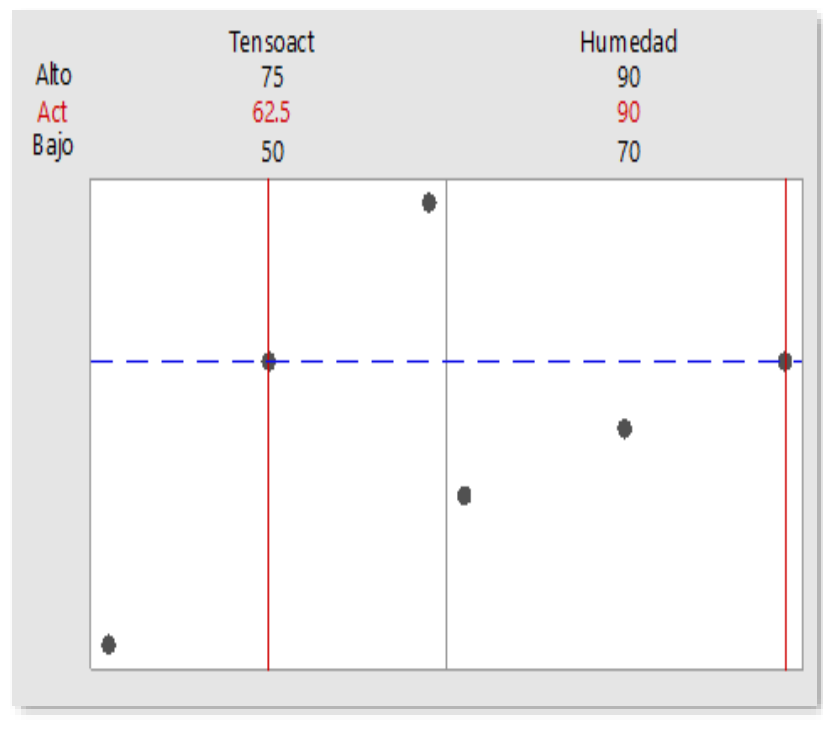

Figura 10 Prueba de varianzas constantes Fuente: Elaboración propia en software Minitab

\section{Mejorar y Controlar}

Una vez obtenidos los resultados de las experimentaciones en donde se indica que sólo los efectos principales son significativos y no su interacción, se procede a establecer condiciones de operación óptimas para el manejo de la piel, dichas condiciones permitirán mejorar la resistencia a la tensión del material en operaciones de ensamble dentro de la industria automotriz. La variable de respuesta (resistencia a la tensión) que se pretende optimizar es del tipo mayor es mejor, y está relacionada de forma significativa a los factores cantidad de tensoactivo y $\%$ de humedad de acuerdo al análisis estadístico mostrado previamente. Para el factor cantidad de tensoactivo es importante establecer un nivel medio $62.5 \mathrm{ml}$, ya que al emplear un nivel alto se pierden propiedades estéticas de la piel y no es técnicamente viable, aunque se registre una mayor resistencia. En el factor porcentaje de humedad es importante que se aplique un porcentaje de humedad del $90 \%$, aunque cabe resaltar que la cantidad de tensoactivo aporta cambios más significativos en la resistencia de la piel que la humedad por lo que no es necesario recurrir a niveles más altos de humedad a partir de una segunda experimentación. De acuerdo con la Figura 11, correspondiente a la gráfica de optimización un valor óptimo de resistencia está situado en un valor de medio de tensoactivo y un nivel alto de humedad con una resistencia promedio estimada de 170.727 Newtons y una estimación por intervalo de $(169.479,171.974)$ con un $95 \%$ de confianza.

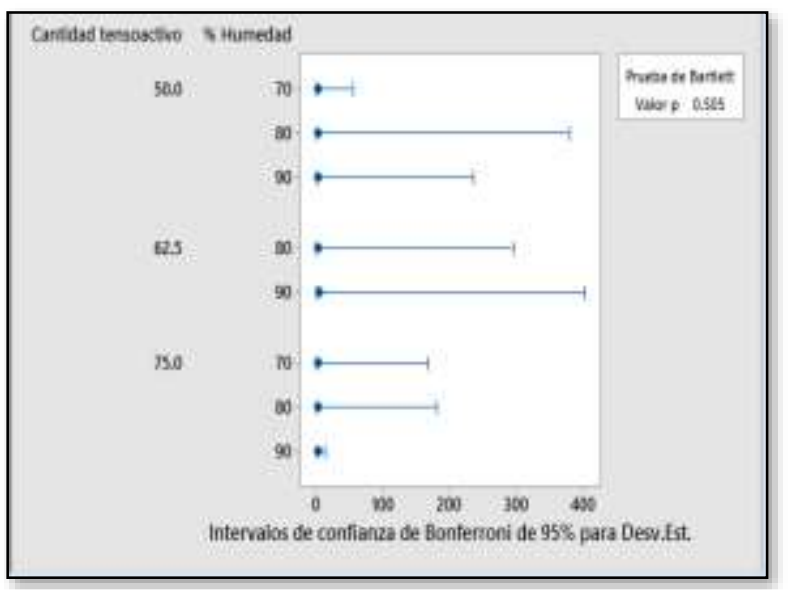

Figura 11 Prueba de varianzas constantes Fuente: Elaboración propia en software Minitab

Las condiciones de operación propuestas a partir del diseño experimental fueron validadas por medio de una muestra de 1000 muestras de piel, de las cuales se detectaron un total de 13 unidades desgarradas (1.3\%). El nivel de sigmas quedo definido en 2.22. El comparativo del antes y después de diseño experimental se muestra en el Gráfico 1.

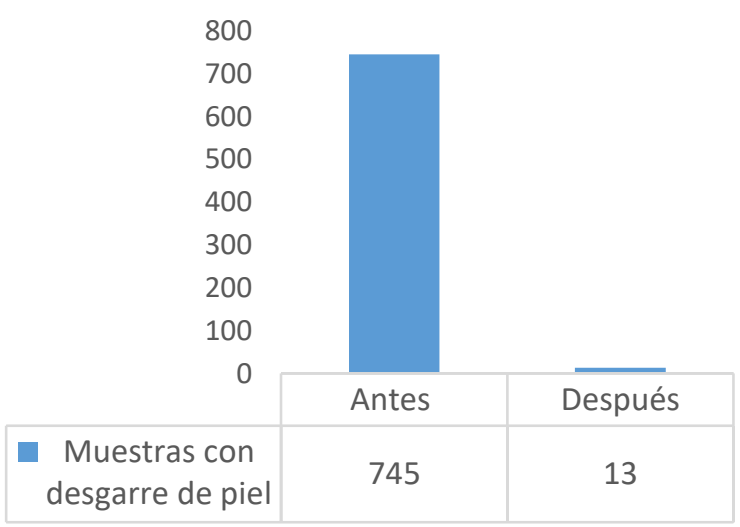

Gráfico 1 Comparación de resultados

Fuente: Elaboración propia

\section{Conclusiones y Discusión}

A partir del presente trabajo de investigación es posible establecer condiciones de operación óptimas para el manejo de la piel en sistemas de producción involucrados en el sector automotriz. De acuerdo a los resultados obtenidos mediante el diseño experimental se validó que el tensoactivo y la humedad son significativos para aumentar la resistencia a la tensión en la piel, los tratamientos y secuencias de experimentación fueron llevados a cabo en el marco de la norma ISO 3376, que especifica el procedimiento y zonas para obtener cada una de las muestras. 
En cuanto al análisis estadístico es importante señalar que no existe un efecto de curvatura en los factores estudiados lo que sugiere establecer niveles altos para este caso, aunque no se debe dejar de lado el aspecto técnico y de calidad en los cuales se contemplan características estéticas o de atributos, por lo que los niveles óptimos quedan definidos con un nivel de tensoactivo medio $(62.5 \mathrm{ml})$ y la humedad en un nivel alto (90\%). En cuanto a la mejora del proceso al implementar las condiciones establecidas por el diseño experimental se mejoraron en un $73.2 \%$ las rasgaduras presentes en la piel, lo cual es sumamente significativo en términos de productividad y economía. El estudio presenta una metodología estadística que cumple con los supuestos establecidos por lo que los resultados son válidos y pueden ser implementados en sistemas productivos que se involucren con piel en el sector automotriz.

\section{Referencias}

Barrera, A., Pulido, A. (2016). La industria automotriz mexicana: situación actual, retos y oportunidades. ISBN: 978-607-97294-2-4

Gryna, F., Chua, T. (2007). Método juran: análisis y planeación de la calidad. McGrawHill Interamericana. ISBN: 9789701061428

Montgomery, D. (2005). Diseño y Análisis de experimentos (2a. ed.). México: Limusa Wiley. ISBN: 9789681861568

Font, G. (2014). Análisis y ensayos en la industria del curtido. Generalitat de Catalunya. McCarty, T. (2004). Six Sigma black belt handbook. McGraw-Hill. ISBN: 9780071501453

Suchana, A., Wu, T., Zhang Y. (2017). Thermomechanical design optimization of conformal cooling channels using design of experiments approach. Procedia manufacturing (10): 898 911.

DOI:

https://doi.org/10.1016/j.promfg.2017.07.078

Tanco, M., Izarbe, L., Viles, E. (2018). Aplicación del Diseño de Experimentos (DOE) para la mejora de procesos.
Romero, J., Queipo, N. (2017). Diseño óptimo basado en metamodelos de un pedal de freno tipo Fórmula SAE. Revista Técnica de la Facultad de Ingeniería Universidad del Zulia (40): 11-15.

Villegas, J. (2013). Aplicación de diseño de experimentos para el análisis de secado de un producto. Innovaciones de negocios (19): 145158.

Yongfan, L. (2017). Research on the optimization design of motorcycle engine based on (DOE) methodology. Procedia Engineering (174): 740-747.

DOI: 